\title{
Odd Triplet Pairing in clean Superconductor/Ferromagnet heterostructures
}

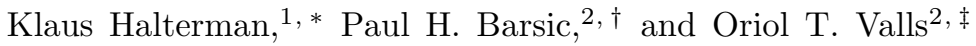 \\ ${ }^{1}$ Physics and Computational Sciences, Research and Engineering Sciences Department, \\ Naval Air Warfare Center, China Lake, California 93555 \\ ${ }^{2}$ School of Physics and Astronomy, University of Minnesota, Minneapolis, Minnesota 55455
}

(Dated: October 27, 2018)

\begin{abstract}
We study triplet pairing correlations in clean Ferromagnet $(\mathrm{F}) /$ Superconductor $(\mathrm{S})$ nanojunctions, via fully self consistent solution of the Bogoliubov-de Gennes equations. We consider FSF trilayers, with $\mathrm{S}$ being an s-wave superconductor, and an arbitrary angle $\alpha$ between the magnetizations of the two F layers. We find that contrary to some previous expectations, triplet correlations, odd in time, are induced in both the $\mathrm{S}$ and $\mathrm{F}$ layers in the clean limit. We investigate their behavior as a function of time, position, and $\alpha$. The triplet amplitudes are largest at times on the order of the inverse "Debye" frequency, and at that time scale they are long ranged in both S and F. The zero temperature condensation energy is found to be lowest when the magnetizations are antiparallel.
\end{abstract}

PACS numbers: 74.45.+c, 74.25.Bt, 74.78.Fk

The proximity effects in superconductor/ferromagnet (SF) heterostructures lead to the coexistence of ferromagnetic and superconducting ordering and to novel transport phenomena [1, 2]. Interesting effects that arise from the interplay between these orderings have potential technological applications in fields such as spintronics 3 . For example, the relative orientation of the magnetizations in the $\mathrm{F}$ layers in FSF trilayers can have a strong influence on the conductivity [4, 5, [6, 7, 8], making them good spin valve candidates. Such trilayers were first proposed [9] for insulating $\mathrm{F}$ layers and later for metallic [10, 11] ones.

This interplay also results in fundamental new physics. An outstanding example is the existence of "odd" triplet superconductivity. This is an s-wave pairing triplet state that is even in momentum, and therefore not destroyed by nonmagnetic impurities, but with the triplet correlations being odd in frequency, so that the equal time triplet amplitudes vanish as required by the Pauli principle. This exotic pairing state with total spin one was proposed long ago [12] as a possible state in superfluid ${ }^{3} \mathrm{He}$. Although this type of pairing does not occur there, it is possible in certain FSF systems [1, 2, 13, 14] with ordinary singlet pairing in S. This arrangement can induce, via proximity effects, triplet correlations with $m=0$ and $m= \pm 1$ projections of the total spin. If the magnetization orientations in both $\mathrm{F}$ layers are unidirectional and along the quantization axis, symmetry arguments show that only the $m=0$ projection along that axis can exist.

Odd triplet pairing in F/S structures has been studied in the dirty limit through linearized Usadel-type quasiclassical equations [2, 13, 14, 15]. In this case, it was found that $m=0$ triplet pairs always exist. They are suppressed in F over short length scales, just as the singlet pairs. The $m= \pm 1$ components, for which the exchange field is not pair-breaking, can be long ranged, and were found to exist for nonhomogeneous magnetization. For FSF trilayers [2, 16, 17], the quasiclassical methods predict that the structure contains a superposition of all

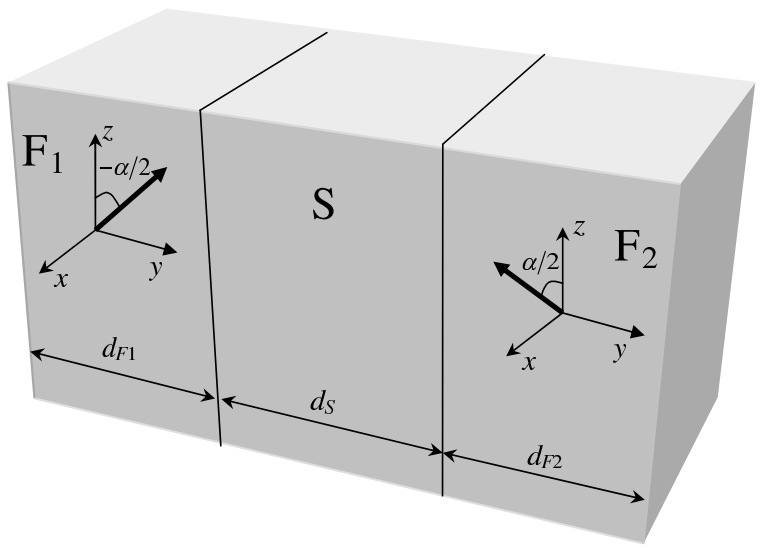

FIG. 1: Schematic of FSF junction. The left ferromagnetic layer $F_{1}$ has a magnetization oriented at an angle $-\alpha / 2$ in the $x-z$ plane, while the other ferromagnet, $\mathrm{F}_{2}$, has a magnetization orientation at an angle $\alpha / 2$ in the $x-z$ plane.

three spin triplet projections except when the magnetizations of the $\mathrm{F}$ layers are collinear, in which case the $m= \pm 1$ components along the magnetization axis vanish. It is noted in Ref. [1] that the existence of such effects in the clean limit has not been established and may be doubted. This we remedy in the present work, where we establish that, contrary to the doubts voiced there, induced, long-ranged, odd triplet pairing does occur in clean FSF structures.

Experimental results that may argue for the existence of long range triplet pairing of superconductors through a ferromagnet have been obtained in superlattices 18 with ferromagnetic spacers, and in two superconductors coupling through a single ferromagnet [19, 20]. Measurements [19] on a SQUID, in which a phase change of $\pi$ in the order parameter is found after inversion, indicate an odd-parity state. Very recently, a Josephson current through a strong ferromagnet was observed, indicating the existence of a spin triplet state [20] induced 
by $\mathrm{NbTiN}$, an s-wave superconductor.

In this paper, we study the induced odd triplet superconductivity in FSF trilayers in the clean limit through a fully self-consistent solution of the microscopic Bogoliubov-de Gennes (BdG) equations. We consider arbitrary relative orientation of the magnetic moments in the two $\mathrm{F}$ layers. We find that there are indeed induced odd triplet correlations which can include both $m=0$ and $m= \pm 1$ projections. We directly study their time dependence and we find that they are largest for times of order of the inverse cutoff "Debye" frequency. The correlations are, at these time scales, long ranged in both the $\mathrm{S}$ and $\mathrm{F}$ regions. We also find that the condensation energy depends on the relative orientation of the F layers, being a minimum when they are antiparallel.

To find the triplet correlations arising from the nontrivial spin structure in our FSF system, we use the BdG equations with the BCS Hamiltonian, $\mathcal{H}_{\text {eff }}$ :

$$
\mathcal{H}_{\mathrm{eff}}=\int d^{3} r\left\{\sum_{\delta} \psi_{\delta}^{\dagger}(\mathbf{r})\left[-\frac{\nabla^{2}}{2 m^{*}}-E_{F}\right] \psi_{\delta}(\mathbf{r})+\frac{1}{2}\left[\sum_{\delta, \beta}\left(i \sigma_{y}\right)_{\delta \beta} \Delta(\mathbf{r}) \psi_{\delta}^{\dagger}(\mathbf{r}) \psi_{\beta}^{\dagger}(\mathbf{r})+\text { h.c. }\right]-\sum_{\delta, \beta} \psi_{\delta}^{\dagger}(\mathbf{r})(\mathbf{h} \cdot \boldsymbol{\sigma})_{\delta \beta} \psi_{\beta}(\mathbf{r})\right\},
$$

where $\Delta(\mathbf{r})$ is the pair potential, to be determined selfconsistently, $\psi_{\delta}^{\dagger}, \psi_{\delta}$ are the creation and annihilation operators with spin $\delta, E_{F}$ is the Fermi energy, and $\boldsymbol{\sigma}$ are the Pauli matrices. We describe the magnetism of the $\mathrm{F}$ layers by an effective exchange field $\mathbf{h}(\mathbf{r})$ that vanishes in the $\mathrm{S}$ layer. We will consider the geometry depicted in Fig. 11 with the $y$ axis normal to the layers and $\mathbf{h}(\mathbf{r})$ in the $x-z$ plane (which is infinite in extent) forming an angle $\pm \alpha / 2$ with the $z$ axis in each $\mathrm{F}$ layer.

Next, we expand the field operators in terms of a Bogoliubov transformation which we write as:

$$
\psi_{\delta}(\mathbf{r})=\sum_{n}\left(u_{n \delta}(\mathbf{r}) \gamma_{n}+\eta_{\delta} v_{n \delta}(\mathbf{r}) \gamma_{n}^{\dagger}\right),
$$

where $\eta_{\delta} \equiv 1(-1)$ for spin down (up), $u_{n \delta}$ and $v_{n \delta}$ are the quasiparticle and quasihole amplitudes. This transformation diagonalizes $\mathcal{H}_{\text {eff }}:\left[\mathcal{H}_{\text {eff }}, \gamma_{n}\right]=-\epsilon_{n} \gamma_{n},\left[\mathcal{H}_{\text {eff }}, \gamma_{n}^{\dagger}\right]=$ $\epsilon_{n} \gamma_{n}^{\dagger}$. By taking the commutator $\left[\psi_{\delta}(\mathbf{r}), \mathcal{H}_{\text {eff }}\right]$, and with $\mathbf{h}(\mathbf{r})$ in the $x-z$ plane as explained above, we have the following:

$$
\begin{aligned}
{\left[\psi_{\uparrow}(\mathbf{r}), \mathcal{H}_{\mathrm{eff}}\right] } & =\left(\mathcal{H}_{\mathrm{e}}-h_{z}\right) \psi_{\uparrow}(\mathbf{r})-h_{x} \psi_{\downarrow}(\mathbf{r})+\Delta(\mathbf{r}) \psi_{\downarrow}^{\dagger}(\mathbf{r}), \\
{\left[\psi_{\downarrow}(\mathbf{r}), \mathcal{H}_{\mathrm{eff}}\right] } & =\left(\mathcal{H}_{\mathrm{e}}+h_{z}\right) \psi_{\downarrow}(\mathbf{r})-h_{x} \psi_{\uparrow}(\mathbf{r})-\Delta(\mathbf{r}) \psi_{\uparrow}^{\dagger}(\mathbf{r}) .
\end{aligned}
$$

Inserting (1) into (2) and introducing a set $\rho$ of Pauli-like matrices in particle-hole space, yields the spin-dependent BdG equations:

$$
\left[\rho_{z} \otimes\left(\mathcal{H}_{0} \hat{\mathbf{1}}-h_{z} \sigma_{z}\right)+\left(\Delta(y) \rho_{x}-h_{x} \hat{\mathbf{1}}\right) \otimes \sigma_{x}\right] \Phi_{n}=\epsilon_{n} \Phi_{n},
$$

where $\Phi_{n} \equiv\left(u_{n \uparrow}(y), u_{n \downarrow}(y), v_{n \uparrow}(y), v_{n \downarrow}(y)\right)^{\mathrm{T}}$ and $\mathcal{H}_{0} \equiv$ $-\partial_{y}^{2} /(2 m)+\varepsilon_{\perp}-E_{F}$. Here $\varepsilon_{\perp}$ is the transverse kinetic energy and a factor of $e^{i \mathbf{k}_{\perp} \cdot \mathbf{r}}$ has been suppressed. In deriving Eq. (3) care has been taken to consistently use the phase conventions in Eq. (1). To find the quasiparticle amplitudes along a different quantization axis in the $x-z$ plane, one performs a spin rotation: $\Phi_{n} \rightarrow \widehat{U}\left(\alpha^{\prime}\right) \Phi_{n}$, where $\widehat{U}\left(\alpha^{\prime}\right)=\cos \left(\alpha^{\prime} / 2\right) \hat{\mathbf{1}} \otimes \hat{\mathbf{1}}-i \sin \left(\alpha^{\prime} / 2\right) \rho_{z} \otimes \sigma_{y}$.

When the magnetizations of the $\mathrm{F}$ layers are collinear, one can take $h_{x}=0$. For the general case shown in Fig. 1 one has in the $\mathrm{F}_{1}$ layer, $h_{x}=h_{0} \sin (-\alpha / 2)$ and $h_{z}=h_{0} \cos (-\alpha / 2)$, where $h_{0}$ is the magnitude of $\mathbf{h}$, while in $\mathrm{F}_{2}, h_{x}=h_{0} \sin (\alpha / 2)$, and $h_{z}=h_{0} \cos (\alpha / 2)$. With an appropriate choice of basis, Eqs. (3) are cast into a matrix eigenvalue system that is solved iteratively with the self consistency condition, $\Delta(y)=g(y) f_{3}$ $\left(f_{3}=\frac{1}{2}\left[\left\langle\psi_{\uparrow}(\mathbf{r}) \psi_{\downarrow}(\mathbf{r})\right\rangle-\left\langle\psi_{\downarrow}(\mathbf{r}) \psi_{\uparrow}(\mathbf{r})\right\rangle\right]\right)$. In the $\mathrm{F}$ layers we have $g(y)=0$, while in S, $g(y)=g, g$ being the usual BCS singlet coupling constant there. Through Eqs. (10), the self-consistency condition becomes a sum over states restricted by the factor $g$ to within $\omega_{D}$ from the Fermi surface. Iteration is performed until self-consistency is reached. The numerical process is the same that was used in previous work [24, 25], with now the $h_{x}$ term requiring larger four-component matrices to be diagonalized.

We now define the following time dependent triplet amplitude functions in terms of the field operators,

$$
\begin{aligned}
& \tilde{f}_{0}(\mathbf{r}, t)=\frac{1}{2}\left[\left\langle\psi_{\uparrow}(\mathbf{r}, t) \psi_{\downarrow}(\mathbf{r}, 0)\right\rangle+\left\langle\psi_{\downarrow}(\mathbf{r}, t) \psi_{\uparrow}(\mathbf{r}, 0)\right\rangle\right], \\
& \tilde{f}_{1}(\mathbf{r}, t)=\frac{1}{2}\left[\left\langle\psi_{\uparrow}(\mathbf{r}, t) \psi_{\uparrow}(\mathbf{r}, 0)\right\rangle-\left\langle\psi_{\downarrow}(\mathbf{r}, t) \psi_{\downarrow}(\mathbf{r}, 0)\right\rangle\right],
\end{aligned}
$$

which, as required by the Pauli principle for these $s$-wave amplitudes, vanish at $t=0$, as we shall verify. Making use of Eq. (11) and the commutators, one can derive and formally integrate the Heisenberg equation of the motion for the operators and obtain:

$$
\begin{aligned}
& \tilde{f}_{0}(y, t)=\frac{1}{2} \sum_{n}\left[u_{n \uparrow}(y) v_{n \downarrow}(y)-u_{n \downarrow}(y) v_{n \uparrow}(y)\right] \zeta_{n}(t), \quad(5 \\
& \tilde{f}_{1}(y, t)=-\frac{1}{2} \sum_{n}\left[u_{n \uparrow}(y) v_{n \uparrow}(y)+u_{n \downarrow}(y) v_{n \downarrow}(y)\right] \zeta_{n}(t),
\end{aligned}
$$




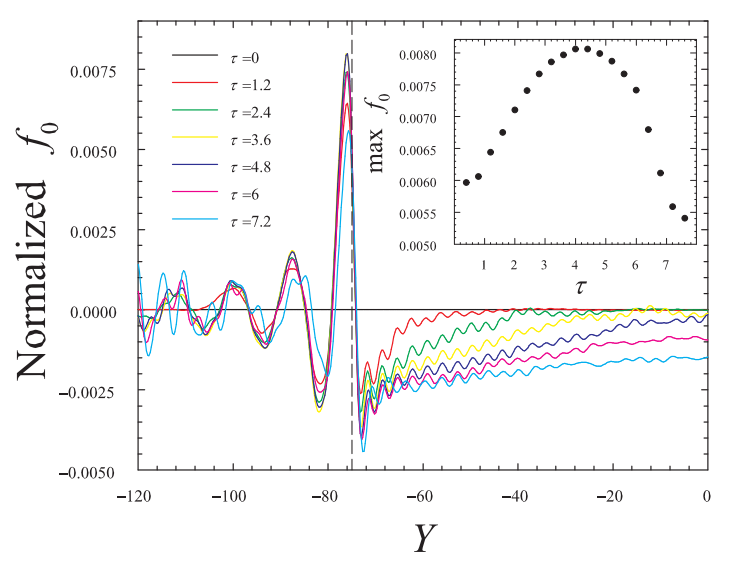

FIG. 2: (Color online) The real part, $f_{0}$, of the triplet amplitude $\tilde{f}_{0}$, for a FSF trilayer at 7 different times. We normalize $f_{0}$ by the singlet bulk pair amplitude, $\Delta_{0} / g$. The coordinate $y$ is scaled by the Fermi wavevector, $Y \equiv k_{F} y$, and time by the Debye frequency, $\tau \equiv \omega_{D} t$. At $\tau=0, f_{0} \equiv 0$ as required by the Pauli principle. The interface is marked by the vertical dashed line, with an $\mathrm{F}$ region to the left and the $\mathrm{S}$ to the right. Half of the $\mathrm{S}$ region and part of the left $\mathrm{F}$ layer are shown. The inset shows the maximum value of $f_{0}$ versus $\tau$.

where $\zeta_{n}(t) \equiv \cos \left(\epsilon_{n} t\right)-i \sin \left(\epsilon_{n} t\right) \tanh \left(\epsilon_{n} / 2 T\right)$.

The amplitudes in Eqs. (5) contain all information on the space and time dependence of induced triplet correlations throughout the FSF structure. The summations in Eqs. (5) are over the entire self-consistent spectrum, ensuring that $f_{0}$ and $f_{1}$ vanish identically at $t=0$ and thus obey the exclusion principle. Using a non self consistent $\Delta(y)$ leads to violations of this condition, particularly near the interface where proximity effects are most pronounced. Geometrically, the indirect coupling between magnets is stronger with fairly thin $\mathrm{S}$ layers and relatively thick $\mathrm{F}$ layers. We thus have chosen $d_{S}=(3 / 2) \xi_{0}$ and $d_{F 1}=d_{F 2}=\xi_{0}$, with the BCS correlation length $\xi_{0}=100 k_{F}^{-1}$. We consider the low $T$ limit and take $\omega_{D}=0.04 E_{F}$. The magnetic exchange is parametrized via $I \equiv h_{0} / E_{F}$. Results shown are for $I=0.5$ (unless otherwise noted) and the magnetization orientation angle, $\alpha$, is swept over the range $0 \leq \alpha \leq \pi$. No triplet amplitudes arise in the absence of magnetism $(I=0)$.

For the time scales considered here, the imaginary parts of $\tilde{f}_{0}(y, t)$ and $\tilde{f}_{1}(y, t)$ at $t \neq 0$ are considerably smaller than their real parts, and thus we focus on the latter, which we denote by $f_{0}(y, t)$ and $f_{1}(y, t)$. In Fig. 2, the spatial dependence of $f_{0}$ is shown for parallel magnetization directions $(\alpha=0)$ at several times $\tau \equiv \omega_{D} t$. The spatial range shown includes part of the $F_{1}$ layer (to the left of the dashed line) and half of the $\mathrm{S}$ layer (to the right). At finite $\tau$, the maximum occurs in the ferromagnet close to the interface, after which $f_{0}$ undergoes damped oscillations with the usual spatial length

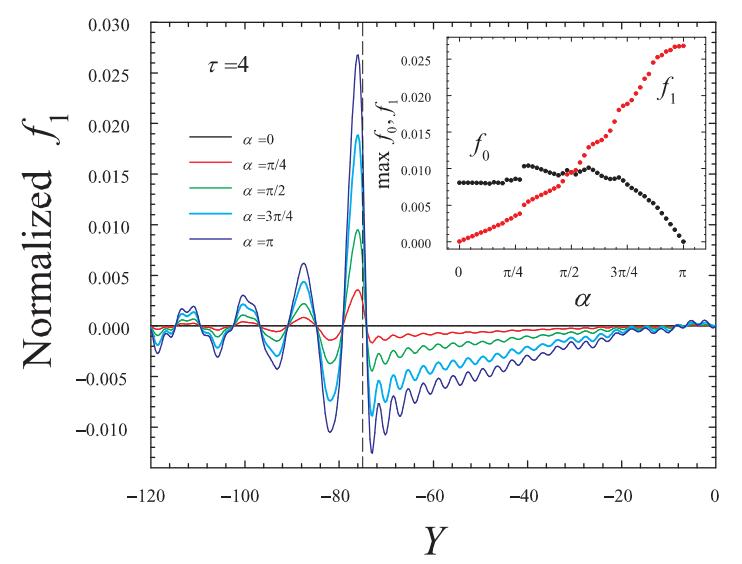

FIG. 3: (Color online) Spatial and angular dependence of $f_{1}$, at $\tau=4 \approx \tau_{c}$ and several $\alpha$. Normalizations and ranges are as in Fig. 2 Inset: maxima of $f_{0}$ and $f_{1}$ in $\mathrm{F}_{1}$ versus $\alpha$.

scale $\xi_{f} \approx\left(k_{F \uparrow}-k_{F \downarrow}\right)^{-1} \approx k_{F}^{-1} / I$. The height of the main peak first increases with time, but drops off after a characteristic time, $\tau_{c} \approx 4$, as seen in the inset, which depicts the maximum value of $f_{0}$ as a function of $\tau$. As $\tau$ increases beyond $\tau_{c}$, the modulating $f_{0}$ in $\mathrm{F}$ develops more complicated atomic scale interference patterns and becomes considerably longer ranged. In $\mathrm{S}$, we see immediately that $f_{0}$ is also larger near the interface. Since the triplet amplitudes vanish at $\tau=0$, short time scales exhibit correspondingly short triplet penetration. The figure shows, however, that the value of $f_{0}$ in $\mathrm{S}$ is substantial for $\tau \gtrsim \tau_{c}$, extending over length scales on the order of $\xi_{0}$ without appreciable decay. In contrast, the usual singlet correlations were found to monotonically drop off from their $\tau=0$ value over $\tau$ scales of order unity.

In the main plot of Fig. 3 we examine the spatial dependence of the real part of the $m= \pm 1$ triplet amplitude, $f_{1}$. Normalizations and spatial ranges are as in Fig. 2 but now the time is fixed at $\tau=4 \approx \tau_{c}$, and five equally spaced magnetization orientations are considered. At $\alpha=0, f_{1}$ vanishes identically at all $\tau$, as expected. For nonzero $\alpha$, correlations in all triplet channels are present. As was found for $f_{0}$, the plot clearly shows that $f_{1}$ is largest near the interface, in the $\mathrm{F}$ region. Our geometry and conventions imply (see Fig. 1) that the magnetization has opposite $x$-components in the $\mathrm{F}_{1}$ and $\mathrm{F}_{2}$ regions. The $f_{1}$ triplet pair amplitude profile is thus antisymmetric about the origin, in contrast to the symmetric $f_{0}$, implying the existence of one node in the superconductor. Nevertheless, the penetration of the $f_{1}$ correlations in $\mathrm{S}$ can be long ranged. We find that $f_{1}$ and $f_{0}$ oscillate in phase and with the same wavelength, regardless of $\alpha$. The inset illustrates the maximum attained values of $f_{0}$ and $f_{1}$ in $\mathrm{F}_{1}$ as $\alpha$ varies. It shows that for 


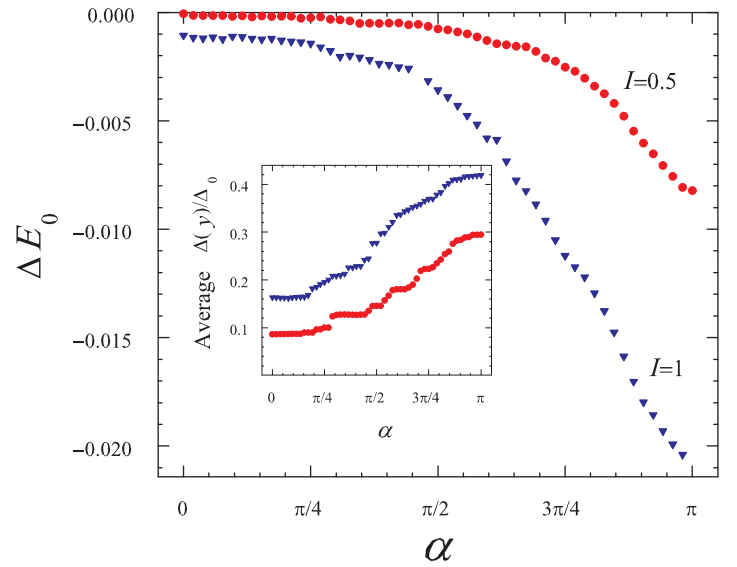

FIG. 4: (Color online) The $T=0$ condensation energy, $\Delta E_{0}$, normalized by $N(0) \Delta_{0}^{2}(N(0)$ is the usual density of states), vs. the angle $\alpha$ for two values of $I$. When the two magnetizations are antiparallel $(\alpha=\pi) \Delta E_{0}$ is lowest. The inset shows the ordinary (singlet) pair potential averaged over the $\mathrm{S}$ region, normalized to the bulk $\Delta_{0}$.

a broad range of $\alpha, \alpha \lesssim 3 \pi / 4$, the maximum of $f_{0}$ varies relatively little, after which it drops off rapidly to zero at $\alpha=\pi$. This is to be expected as the anti-parallel orientation corresponds to the case in which the magnetization is in the $x$ direction, which is perpendicular to the axis of quantization (see Fig. 1). The rise in the maximum of $f_{1}$ is monotonic, cresting at $\alpha=\pi$, consistent with the main plot. At this angle the triplet correlations extend considerably into the superconductor. At $\alpha=\pi / 2$ the maxima coincide since the two triplet components are then identical throughout the whole space because the magnetization vectors have equal projections on the $x$ and $z$ axes. At $\alpha=\pi$ both magnetizations are normal to the axis of quantization $z$ (see Fig. 1). By making use of the rotation matrix $\widehat{U}$ (see below Eq. 3) one can verify that the $m= \pm 1$ components with respect to the axis $x$ along the magnetizations are zero.

We next consider the condensation energy, $\Delta E_{0}$, calculated by subtracting the zero temperature superconducting and normal state free energies. The calculation uses the self consistent spectra and $\Delta(y)$, and methods explained elsewhere 25, 26]. In the main plot of Fig. 4 we show $\Delta E_{0}$ (normalized at twice its bulk $\mathrm{S}$ value) at two different values of $I$. The condensation energy results clearly demonstrate that the antiparallel state $(\alpha=\pi)$ is in general the lowest energy ground state. These results are consistent with previous studies [8] of FSF structures with parallel and antiparallel magnetizations. The inset contains the magnitude of the spatially averaged pair potential, normalized by $\Delta_{0}$, at the same values of $I$. The inset correlates with the main plot, as it shows that the singlet superconducting correlations in $\mathrm{S}$ increase with $\alpha$ and are larger at $I=1$ than at $I=0.5$. The halfmetallic case of $I=1$ illustrates that by having a single spin band populated at the Fermi surface, Andreev reflection is suppressed, in effect keeping the superconductivity more contained within S.

Thus, we have shown that in clean FSF trilayers induced odd triplet correlations, with $m=0$ and $m= \pm 1$ projections of the total spin, exist. We have used a microscopic self-consistent method to study the time and angular dependence of these triplet correlations. The correlations in all 3 triplet channels were found, at times $\tau \equiv \omega_{D} t \gtrsim \tau_{c}$, where $\tau_{c} \approx 4$, to be long ranged in both the $\mathrm{F}$ and $\mathrm{S}$ regions. Finally, study of the condensation energy revealed that the ground state energy is always lowest for antiparallel magnetizations.

This project was supported in part by a grant of HPC resources from the ARSC at the University of Alaska Fairbanks (part of the DoD HPCM program) and by the University of Minnesota Graduate School.

* Electronic address: klaus.halterman@navy.mil

$\dagger$ Electronic address: barsic@physics.umn.edu

‡ Electronic address: otvalls@umn.edu Also at Minnesota Supercomputer Institute, University of Minnesota, Minneapolis, Minnesota 55455

[1] A.I. Buzdin, Rev. Mod. Phys. 77, 935 (2005).

[2] F.S. Bergeret, A.F Volkov, and K.B. Efetov, Rev. Mod. Phys. 77, 1321 (2005).

[3] Igor Zutić, Jaroslav Fabian, S. Das Sarma, Rev. Mod. Phys. 76, 323 (2004).

[4] J. Y. Gu et al., Phys. Rev. Lett. 89, 267001 (2002).

[5] I. C. Moraru, W. P. Pratt, N. O. Birge, Phys. Rev. Lett. 96, 037004 (2006).

[6] C. Bell, S. Turşucu, and J. Aarts, Phys. Rev. B 74, 214520 (2006).

[7] C. Visani et al., Phys. Rev. B 75, 054501 (2007).

[8] K. Halterman and O.T. Valls, Phys. Rev. B 72, 060514(R), (2005).

[9] P. G. de Gennes, Phys. Lett. 23, 10 (1966).

[10] L.R. Tagirov, Phys. Rev. Lett. 83, 2058 (1999).

[11] A.I. Buzdin, A.V. Vdyayev, and N.V. Ryzhanova, Europhys. Lett. 48, 686 (1999).

[12] V.L. Berezinskii, JETP Lett. 20, 287, (1974).

[13] F.S. Bergeret, A.F Volkov, and K.B. Efetov, Phys. Rev. Lett. 86, 3140 (2001).

[14] F. S. Bergeret, A. F. Volkov, and K. B. Efetov, Phys. Rev. B 68, 064513 (2003).

[15] T. Champel and M. Eschrig, Phys. Rev. B 72, 054523 (2005).

[16] T. Löfwander et al., Phys. Rev. Lett. 95, 187003 (2005).

[17] Ya. V. Fominov, A. A. Golubov, and M. Yu. Kupriyanov, JETP Lett. 77, 510 (2003).

[18] V. Peña, et al., Phys. Rev. B 69, 224502 (2004).

[19] K. D. Nelson et al., Science 306, 1151 (2004).

[20] R. S. Keizer et al., Nature 439, 825, (2006).

[21] P.G. de Gennes, Superconductivity of Metals and Alloys (Addison-Wesley, Reading, MA, 1989).

[22] A. F. Volkov, F. S. Bergeret, and K. B. Efetov, Phys. 
Rev. Lett. 90, 117006 (2003).

[23] Superconductivity by J. B. Ketterson and S. N. Song (1999), p. 286.

[24] K. Halterman and O.T. Valls, Phys. Rev. B 69, 014517 (2004).
[25] K. Halterman and O.T. Valls, Phys. Rev. B 70, 104516 (2004).

[26] I. Kosztin et al., Phys. Rev. B 58, 9365 (1998). 